\title{
Protein convertase subtilisin/Kexin type 9 inhibits hepatocellular carcinoma growth by interacting with GSTP1 and suppressing the JNK signaling pathway
}

\author{
Mingyan He \\ First Affiliated Hospital of Nanchang University \\ Jing Hu \\ Jiangxi Provincial People's Hospital \\ Tingting Fang \\ Zhongshan Hospital Fudan University \\ Wenqing Tang \\ Zhongshan Hospital Fudan University \\ Bei LV \\ Zhongshan Hospital Fudan University \\ Biwei Yang \\ Zhongshan Hospital Fudan University \\ Jinglin Xia ( $\nabla$ xiajinglin_fd@sina.com )
}

\section{Research}

Keywords: Hepatocellular carcinoma, protein convertase subtilisin/Kexin type 9, glutathione S-transferase Pi 1, Jun N-terminal kinase

Posted Date: June 10th, 2020

DOI: https://doi.org/10.21203/rs.3.rs-32718/v1

License: (a) This work is licensed under a Creative Commons Attribution 4.0 International License. Read Full License 


\section{Abstract}

Background PCSK9 has been found to be closely related to the occurrence and development of a variety of tumors. However, the concrete role of PCSK9 and its relationship with HCC development is largely unknown.

Methods The expression levels of PCSK9 in HCC tissues and HCC cell lines were determined by quantitative real-time polymerase chain reaction (qRT-PCR), western blot and immunohistochemistry assays. The effects of PCSK9 expression on HCC biological traits were investigated by overexpressing and downregulating PCSK9 protein in vivo and in vitro. The mechanism by which PCSK9 mediates the depolymerization of the GSTP1 dimer and the phosphorylation of the JNK signaling pathway was further investigated.

Results PCSK9 is downregulated in human HCC tissues. HCC cell proliferation, cell cycle phase distribution and apoptosis were inhibited by PCSK9 in vitro. PCSK9 suppresses tumor growth and metastasis of HCC in vivo. PCSK9 interacts with GSTP1 and promotes the depolymerization of the GSTP1 dimer and inactivation of the JNK signaling pathway. Furthermore, low PCSK9 protein expression in primary HCC tissues correlated with worse clinical outcomes.

Conclusions PCSK9 inhibits HCC cell proliferation, cell cycle phase distribution and apoptosis by interacting with GSTP1 and suppressing the JNK signaling pathway, suggesting that PCSK9 might act as a tumor suppressor and may serve as a therapeutic target for the treatment of HCC.

\section{Background}

Hepatocellular carcinoma (HCC) is one of the most frequent malignant tumors and is characterized by an insidious onset, rapid progression, and high mortality rates. In recent years, the management of hepatitis virus carriers and therapeutic advances, including curative hepatectomy, liver transplantation, local treatment, radiation, and molecular targeted therapy, have provided great benefits to patients with HCC. However, the fiveyear survival of small HCC and advanced HCC is still approximately $60 \%$ and $10 \%$, respectively. High aggressiveness, metastasis and recurrence are the key points of poor prognosis. Therefore, it is critical to elucidate the molecular mechanisms underlying HCC progression and develop novel treatment strategies.

Protein convertase subtilisin/Kexin type 9 (PCSK9) is a member of the family of pre protein invertases, which encode secretory serine proteases composed of a signal peptide, pre domain, catalytic domain and C-terminal rich cysteine histidine carboxyl domain. Its well-known function is to regulate the posttranscriptional degradation of LDLR (low-density lipoprotein receptor) protein by binding with LDLR and promoting LDLR translocation to acidic organelle endosomes or lysosomes. PCSK9 promotes the degradation of LDLR, which leads to a decrease in the cholesterol uptake ability of cells, an increase in LDL-C levels and hypercholesterolemia. In addition, PCSK9 has been reported to play an important role in a variety of biological processes, including the inflammatory response, immune response, metabolism, cell cycle, and apoptosis[1-3]. A previous study reported that PCSK9 expression in HCC tissues is lower than that in paracancerous tissues[4], and our previous study found that Chinese kiwifruit root extract had the effect of inhibiting the development of hepatocarcinoma cells, and PCSK9 is the key molecule of the Chinese herbal extract to inhibit the proliferation of liver cancer cells[5]. In addition, PCSK9 has been found to be closely related to the 
occurrence and development of a variety of tumors, including melanoma, prostate cancer, and lung cancer. [68] However, the concrete role of PCSK9 and its relationship with HCC development is largely unknown.

In this study, we investigated the expression of PCSK9 in HCC cell lines and tumor samples. We explored how HCC growth was related to changes in PCSK9 expression in vitro and in vivo. Using tissue microarrays (TMAs) in HCC samples, we determined the relationship between PCSK9 expression and clinicopathological parameters to evaluate its prognostic significance.

\section{Methods}

\section{Tissue specimens and tissue microarray (TMA)}

mRNA and protein samples of human HCC tissues $(n=48)$ and matched adjacent nontumor samples $(n=48)$ were collected from patients who had undergone curative hepatectomy at Zhongshan Hospital Affiliated with Fudan University.

Preparation of TMA was performed as described[9]. Primary HCC samples for TMA were obtained from 241 postoperative patients with HCC whose 10-year follow-up data were available at Zhongshan Hospital Affiliated with Fudan University. The study was approved by the Zhongshan Hospital Research Ethics Committee. Follow-up procedures were described previously[9].

\section{Immunohistochemistry assays}

The slides were probed with primary antibodies against PCSK9 (1:100, Abcam, Cambridge, MA, USA) and then incubated with horseradish peroxidase-conjugated IgG (1:500; Invitrogen), and the proteins in situ were visualized with 3,3'-diaminobenzidine. The staining extent was determined as previously reported[10].

\section{Cell culture}

The human HCC cell lines HepG2 and Huh7 were purchased from the Cell Bank of the Institute of Biochemistry and Cell Biology, China Academy of Sciences, Shanghai, China. The HCC cell lines MHCC-97H and MHCC-97L were established at the Liver Cancer Institute[11]. Cells were cultured in Dulbecco's modified Eagle's medium (DMEM) supplemented with $10 \%(\mathrm{v} / \mathrm{v}$ ) fetal bovine serum (Gibco, BRL, USA), $10 \mu \mathrm{g} / \mathrm{mL}$ streptomycin sulfate and $100 \mu \mathrm{g} / \mathrm{mL}$ penicillin $\mathrm{G}$. All cells were incubated at $37^{\circ} \mathrm{C}$ in a humidified atmosphere containing $5 \% \mathrm{CO}_{2}$.

\section{Total RNA isolation and quantitative real-time quantitative PCR}

Total RNA was isolated from HCC cells and HCC tissues with TRIzol reagent (Invitrogen, CA, USA) according to the manufacturer's protocol. Complementary DNA was synthesized using the Reverse Transcription Reagent 
Kit (TaKaRa, Shiga, Japan) from 500 ng of RNA. All primers are shown in Supplementary Table 1. Real-time qRT-PCR analysis was performed using an ABI7500 real-time fluorescent measurement system (Applied Biosystems, USA) and a PCR amplification kit (TaKaRa, Japan). Values were derived from at least three independent experiments performed in duplicate and normalized to $\beta$-actin expression. The 2- $\triangle \triangle \mathrm{Ct}$ method was used for the data analysis[12].

\section{Western blot}

The procedures were performed as described[13]. Preparation of nonreducing PAGE gel and protein sample was as follows: preparation of the nonreducing gel was according to the second part of the concentrated rubber and separation gel preparation scheme, without adding SDS. After the protein was extracted, it was diluted with $5 \times$ nondenaturing and nonreducing buffer in a 4:1 ratio without boiling and directly added to the sample hole of the nonreducing PAGE gel. The antibodies used for western blotting included PCSK9 and GSTP1 (Abcam, Cambridge, MA, USA).

\section{Cell viability assays}

HCC cell viability was assessed using CCK-8 assays (Cell Counting Kit-8, Dojindo, Japan). The procedures were performed as described[5].

\section{Cell cycle analysis and Annexin V apoptosis assay}

The procedures were performed as described[14].

\section{Coimmunoprecipitation (Co-IP) and mass spectrometry (IP/MS)}

The procedures were performed as described[10, 15]. The antibodies used in Co-IP included anti-PCSK9 mAb, anti-GSTP1 mAb, and anti-IgG (Abcam, Cambridge, MA, USA).

\section{Immunofluorescence staining}

HCC cells grown on coverslips were fixed with $3 \%$ paraformaldehyde at $4^{\circ} \mathrm{C}$ overnight. To block the nonspecific binding of antibodies, cells were then incubated with $3 \%$ bovine serum albumin for $30 \mathrm{~min}$ at room temperature. After that, cells were incubated with primary antibodies against PCSK9 (1:100, Abcam, Cambridge, MA, USA) and GSTP1 (1:100, Abcam, Cambridge, MA, USA) at $4^{\circ} \mathrm{C}$ overnight. Cells were then probed with secondary antibodies and incubated at room temperature for another $2 \mathrm{~h}$. Cells were then 
mounted with ProLong gold antifade reagent with 4',6-diamidino-2-phenylindole (Life Technologies) and immediately observed under a fluorescence microscope.

\section{Nude mice and metastasis assay}

HCC cells $\left(1.2 \times 10^{8}\right.$ cells in $0.1 \mathrm{ml}$ of phosphate-buffered saline) were injected subcutaneously into the dorsal left flank of eight-week-old male Balb/c nude mice. These mice were randomly divided into four groups before injection. After the tumors were visible, the tumor size was measured every week until 4 weeks. The nude mice were killed, and the subcutaneous tumor was stripped and cut into tumor tissue blocks of $1 \mathrm{~mm} \times 1 \mathrm{~mm} \times$ $\mathrm{mm}$ size as the tumor source for subsequent orthotopic liver planting. The mice inoculated with tumor blocks were sacrificed after eight weeks, and the number of metastatic tumors was assessed by double-blinded evaluation.

\section{Statistical analysis}

Statistical analysis was performed with SPSS 19.0 statistical software (SPSS Inc., Chicago, IL, USA). KaplanMeier analysis was used for survival analysis, and the log-rank test was chosen to compare the differences. The Pearson $\chi 2$ test or Fisher's exact test was employed to compare qualitative variables, while Student's $t$ tests were used for quantitative variables. A Cox proportional hazards model was adopted for multivariate analysis. The level of significance was set at $P<0.05$ for all tests.

\section{Results}

\section{PCSK9 is downregulated in human HCC tissues}

We first determined the PCSK9 mRNA and protein levels in 48 cases of human HCC tissues and in adjacent nontumor samples by qRT-PCR, western blot analysis and immunohistochemistry. PCSK9 expression was decreased in most (34 vs. 14) HCC samples relative to nontumor samples (Fig. 1A, 1B and 1C). PCSK9 expression was further assessed in various $\mathrm{HCC}$ cell lines with different metastatic potentials (HCCLM3, MHCC97H, MHCC97L, Hep3B, HepG2, and Huh7) and the immortal normal liver cell line (HL-7702) (Fig. 1D and 1E). We found that the expression of PCSK9 was lower in the LM3 and 97H cell lines with relatively high malignancy than in the HepG2 and Huh7 cell lines with relatively low malignancy and immortalized hepatocyte lines. These results suggest that PCSK9 is downregulated in the process of hepatocarcinogenesis and development.

\section{PCSK9 suppresses HCC cell proliferation, cell cycle phase distribution and apoptosis in vitro}

To elucidate the biological function of PCSK9 in HCC development, lentivirus-mediated overexpression and knockdown of PCSK9 in HCC cells was performed. The expression of PCSK9 is relatively low in 97H cells and high in HepG2 cells. Therefore, we conducted stable PCSK9 overexpression in 97H cells (97H-PCSK9) and successful knockdown of PCSK9 in HepG2 cells (HepG2-shRNA-PCSK9) (Fig. 2A). 
The proliferation of transfected cells was investigated using a CCK-8 kit. High expression of PCSK9 in $97 \mathrm{H}$ cells was associated with a slower growth rate compared to the respective control, whereas cells transfected with anti-PCSK9 shRNA grew faster than the control in HepG2 cells (Fig. 2B). We further determined whether the altered growth rate is associated with the cell cycle and cell apoptosis. PI staining showed that the cell cycle was arrested in G1 phase, with $66.6 \%$ of $97 \mathrm{H}$-PCSK9 cells in G0/G1 versus $51.9 \%$ of control cells (P $<0.05$ ) (Fig. 2C). In contrast, PCSK9 knockdown in HepG2 cells significantly promoted the cell cycle, with 46.6\% HepG2-shRNA-PCSK9 cells in G0/G1 versus $56.6 \%$ of control cells $(\mathrm{P}<0.05)$ (Fig. $2 \mathrm{C}$ ). Annexin V/PI assays showed a significant apoptosis rate increase in $97 \mathrm{H}-\mathrm{PCSK} 9$ cell lines compared to the respective control, whereas HepG2-shRNA-PCSK9 cells showed a lower apoptosis rate than that of the control (Fig. 2D). In addition, we detected the expression levels of proliferation markers, apoptosis markers and cell cycle proteins. Western blot analysis showed that overexpression of PCSK9 significantly inhibited the expression of proliferating cell nuclear antigen (PCNA), cyclin D1, cyclin D3 and Bcl-2 and increased the expression of Bax, Bid and cleaved caspase-3, whereas PCSK9 knockdown resulted in the opposite effect (Fig. 2E).

\section{Effects of PCSK9 on tumor growth and metastasis of HCC in vivo}

97H cells stably expressing PCSK9 or control vector were injected subcutaneously into nude mice. Palpable tumors formed within 2 weeks. Tumor volume was measured each week, and mice were sacrificed 5 weeks after tumor cell implantation. The average tumor volume of $97 \mathrm{H}$ cells stably transfected with PCSK9 was $0.902 \pm 0.081 \mathrm{~cm}^{3}$, which was significantly smaller than tumors in the control group $\left(1.572 \pm 0.102 \mathrm{~cm}^{3} ; \mathrm{P}<\right.$ 0.01) (Fig. 3A). The average tumor volume of HepG2 cells stably transfected with shRNA-PCSK9 was $0.815 \pm 0.068 \mathrm{~cm}^{3}$, which was significantly smaller than the tumors in the control group $\left(0.407 \pm 0.032 \mathrm{~cm}^{3} ; \mathrm{P}<\right.$ 0.01) (Fig. 3A).

To further determine the effects of PCSK9 on tumor growth and metastasis, we isolated the tumor xenografts above and implanted them into the livers of nude mice to establish orthotopic models. After 8 weeks, the average volume of $97 \mathrm{H}-\mathrm{PCSK} 9$ orthotopic tumors was noticeably smaller than that of the control group (Fig. 3B). In addition, the total number of lung metastasis lesions in the $97 \mathrm{H}$-PCSK9 group was much lower than that in the control group (Fig. 3C). These results suggested that PCSK9 appears to act as a tumor suppressor.

\section{PCSK9 interacts with GSTP1}

To explore the mechanism underlying PCSK9 inhibition of HCC cell development, IP/MS was conducted to identify key factors associated with PCSK9 in $97 \mathrm{H}$ cells transfected with the PCSK9 vector and Huh7 cells with naturally high levels of PCSK9. The PCSK9 protein complex was immunoprecipitated by anti-PCSK9 $\mathrm{mAb}$, with anti-lgG as a control to rule out nonspecific precipitation. The coprecipitated proteins were visualized by silver staining after electrophoresis and subjected to amino acid sequence identification by mass spectrometry analysis. One identified factor turned out to be glutathione S-transferase Pi 1 (GSTP1) (Fig. 4A). To validate the interaction between PCSK9 and GSTP1, coimmunoprecipitation using GSTP1 antibody was also performed, suggesting that PCSK9 could in turn coimmunoprecipitate with GSTP1, as revealed by western blotting (Fig. 4B). Similar results were validated in Huh7 cells (Fig. 4D). Moreover, coexpressed PCSK9 and GSTP1 were found to colocalize in 97H-PCSK9 cells (Fig. 4C). 
PCSK9 promotes the depolymerization of the GSTP1 dimer and inactivation of the JNK signaling pathway

GSTP1 plays an important role in the regulation of the Jun N-terminal kinase (JNK) signaling pathway and participates in cell proliferation, the cell cycle, and apoptosis, among other processes. To determine the role of GSTP1 in the tumor-suppressive property of PCSK9, we detected the protein expression of GSTP1 and JNK signaling activity. No significant differences in the expression level of GSTP1 were found, whereas phosphorylated JNK and phosphorylated c-Jun were downregulated by the overexpression of PCSK9 (Fig. $5 \mathrm{~A})$. Previous studies have shown that monomer GSTP1 has a higher activity of binding JNK protein than dimer GSTP1 and can inhibit the phosphorylation of JNK protein and its downstream signaling pathway activity[16]. We found that the level of GSTP1 dimer in 97H-PCSK9 cells was significantly lower than that in 97H-mock cells, while the content of GSTP1 monomer was significantly increased, indicating that PCSK9 overexpression can promote the depolymerization of GSTP1 dimer (Fig. 5B). We further examined the binding of GSTP1 to JNK and found a significantly higher level of JNK protein coprecipitated by anti-GSTP1 in 97HPCSK9 cells than in 97H-mock cells (Fig. 5C), suggesting that PCSK9 protein promotes the transformation of GSTP1 from dimer to monomer and enhances the binding ability of GSTP1 with JNK protein.

\section{Low PCSK9 protein expression in primary HCC tissues correlated with worse clinical outcomes}

$\mathrm{IHC}$ on a tissue microarray was performed to measure PCSK9 protein levels in HCC samples from 241 postoperative patients with HCC whose 10-year follow-up data were available. Except for 6 samples damaged during array preparation, the remaining 235 samples were analyzed. All HCC samples could be stratified into either the high PCSK9 level (PCSK9-hi) or low PCSK9 level (PCSK9-lo) groups according to IHC staining scores. Comparisons of clinicopathologic profiles between patients with HCC with PCSK9-hi and PCSK9-lo are shown in Supporting Table S1. The PCSK9-lo group displayed significantly worse overall survival (OS) (median OS: 64.2 months versus 83.2 months; Log-rank=4.237, $p=0.04$ ) and recurrence-free survival (RFS) (median RFS: 26.5 months versus 46.6 months; Log-rank=10.498, $p=0.001$ ) compared to the PCSK9-hi group (Fig. 6).

A high AFP level, high CA199 level, BCLC stage, and absent tumor encapsulation were found to be associated with worse OS and RFS in univariate analysis (Table 1). To further assess the correlation between the PCSK9 level and other risk factors, a Cox proportional hazards analysis was performed, which indicated that a low PCSK9 level was an independent risk factor for worse OS (hazard ratio=2.025, 95\% Cl: 1.384-2.961, p=0.000) and shortened RFS (hazard ratio=1.749, 95\% Cl: 1.034-2.958, $p=0.037$ ) (Table 1).

\section{Discussion}

In this study, we demonstrated that PCSK9 was expressed at a lower level in HCC tissues than in adjacent nontumor samples. In vivo and in vitro experiments suggested that PCSK9 inhibited HCC cell proliferation and lung metastasis. Further analysis showed that PCSK9 interacts with GSTP1 and promotes the depolymerization of the GSTP1 dimer and inactivation of the JNK signaling pathway. Moreover, a positive correlation between PCSK9 protein expression and clinical outcomes was validated. These data suggest that PCSK9 might act as a tumor suppressor in HCC. 
Previous studies reported that the PCSK9 protein can induce the degradation of many lipid-related protein receptors and other proteins, including low-density lipoprotein receptor (LDLR), very-low-density lipoprotein receptor (VLDLR)[17], apoE receptor 2[17], CD36 protein[18], and CD81[19]. It has also been reported that PCSK9 is related to various biological pathways, such as the inflammatory response, immune response, metabolism, cell cycle and apoptosis[20-22], suggesting that PCSK9 is a multifunctional protein. However, only a few studies have reported the relationship between PCSK9 and cancer[6, 8]. One study on HCC reported a decrease in PCSK9 expression in human HCC tissues but did not clarify the specific significance of low PCSK9 expression levels in the carcinogenesis and development of HCC[4]. Our study has demonstrated, for the first time, that PCSK9 can interact with GSTP1, affect JNK phosphorylation levels, and inhibit HCC proliferation, which is of great significance for further understanding the function of the PCSK9 protein and suggests that PCSK9 may serve as a therapeutic target for patients with HCC. Additionally, whether the function of PCSK9, as an important gene regulating the intracellular transport and degradation of LDLR membrane protein, is affected by GSTP1 protein has not been reported. Further study on the effect of GSTP1 on the PCSK9/LDLR signaling pathway is of great significance for further understanding cell cholesterol metabolism.

GSTP1 is an important subtype of human GSTs that can reduce the excessive ROS and oxygen free radicals produced in organisms through catalytic mechanisms and maintain the balance of oxidation-reduction in organisms. GSTP1 also regulates the MAPK-dependent cell cycle, proliferation, apoptosis, the inflammatory response, DNA damage repair and other biological processes through ligand binding properties[23-24]. JNKs are members of the mitogen-activated protein kinase (MAPK) family. Under the pressure or stimulation of reactive oxygen species, radiation, inflammatory factors, growth factors, etc., JNK serine/tyrosine kinase activity is activated, which promotes the phosphorylation of downstream substrates (such as c-Jun, c-Fos, and ATF2) and further promotes the formation of an activated protein-1 (AP-1) transcription complex[25-26]. In the tumor environment, cancer cells undergo proto-oncogene transformation, and the JNK signaling pathway is usually activated and plays an important role in carcinogenesis and progression[27-29]. GSTP1 is a natural inhibitor of the JNK signaling pathway through binding to the carboxyl terminal negative charge region of the JNK protein[14, 16, 30]. Compared with the GSTP1 dimer, the GSTP1 monomer has higher JNK protein binding activity. While cells are stimulated by reactive oxygen species, chemicals, inflammatory factors, etc., GSTP1 crosslinks to form dimers or polymers, relieving the inhibition of JNK and activating the JNK signaling pathway[31]. Increased expression of GSTP1 has been found in most tumor tissues and is closely related to tumorigenesis and development. In the present study, PCSK9 interacts with GSTP1 to affect JNK signaling pathway activity and thus affects the biological function of HCC cells, which is consistent with previous studies of GSTP1. On the other hand, our results showed that the activation of GSTP1 is also regulated by the PCSK9 protein, which opens up a way for us to further explore the functions of GSTP1.

In conclusion, the collective findings from our study show for the first time that PCSK9 acts as a tumor suppressor gene in HCC. The mechanistic link among PCSK9, GSTP1, and JNK signaling indicates that PCSK9 is a potential therapeutic target for HCC development and metastasis.

\section{List Of Abbreviations}


Hepatocellular carcinoma (HCC), protein convertase subtilisin/Kexin type 9 (PCSK9), low-density lipoprotein receptor (LDLR), very-low-density lipoprotein receptor (VLDLR), glutathione S-transferase Pi 1 (GSTP1), Jun Nterminal kinase (JNK), mitogen activated protein kinases (MAPKs), activated protein-1 (AP-1), tissue microarrays (TMAs), overall survival (OS), recurrence-free survival (RFS), proliferating cell nuclear antigen (PCNA).

\section{Declarations}

\section{Ethics approval and consent to participate}

Ethical approval for the study was provided by the ethics committee of Zhongshan Hospital Affiliated with Fudan University. Written informed consent was obtained from all subjects or their guardians.

\section{Consent for publication}

Informed consent for publication was obtained from all subjects or their guardians.

\section{Availability of data and materials}

Not applicable.

\section{Competing interests}

All authors have no conflicts of interest to disclose.

\section{Funding}

This study was sponsored by grants from the National Natural Science Foundation of China (No. 81802461) and the Science \& Technology Innovation Talent Project of Jiangxi Provincial Education Department (No. GJJ170010).

\section{Authors' contributions}

JLX conceived and designed the study. MYH and JH performed the experiments. TTF, WQT, BL, BWY analyzed the data. All authors read and approved the final manuscript.

\section{Acknowledgments}

Not applicable.

\section{References}

1. KR Feingold, AH Moser, JK Shigenaga, SM Patzek, C Grunfeld. Inflammation stimulates the expression of PCSK9. BIOCHEM BIOPH RES CO. 2008;374(2):341-44.

2. KR Walley, KR Thain, JA Russell, MP Reilly, NJ Meyer, JF Ferguson,et al. PCSK9 is a critical regulator of the innate immune response and septic shock outcome. SCI TRANSL MED. 2014;6(258):143r-258r. 
3. H Lan, L Pang, MM Smith, D Levitan, W Ding, L Liu,et al. Proprotein convertase subtilisin/kexin type 9 (PCSK9) affects gene expression pathways beyond cholesterol metabolism in liver cells. J CELL PHYSIOL. 2010:n/a-n/a.

4. M Bhat, N Skill, V Marcus, M Deschenes, X Tan, J Bouteaud,et al. Decreased PCSK9 expression in human hepatocellular carcinoma. BMC GASTROENTEROL. 2015;15(1).

5. M He, J Hou, L Wang, M Zheng, T Fang, X Wang,et al. Actinidia chinensis Planch root extract inhibits cholesterol metabolism in hepatocellular carcinoma through upregulation of PCSK9. Oncotarget. 2017.

6. X Xu, Y Cui, L Cao, Y Zhang, Y Yin, X Hu. PCSK9 regulates apoptosis in human lung adenocarcinoma A549 cells via endoplasmic reticulum stress and mitochondrial signaling pathways. EXP THER MED. 2017;13(5):1993-99.

7. X Sun, R Essalmani, R Day, AM Khatib, NG Seidah, A Prat. Proprotein convertase subtilisin/kexin type 9 deficiency reduces melanoma metastasis in liver. NEOPLASIA. 2012;14(12):1122-31.

8. SS Gan, JQ Ye, L Wang, FJ Qu, CM Chu, YJ Tian,et al. Inhibition of PCSK9 protects against radiationinduced damage of prostate cancer cells. Onco Targets Ther. 2017;10:2139-46.

9. JB Jia, WQ Wang, HC Sun, XD Zhu, L Liu, PY Zhuang,et al. High expression of macrophage colonystimulating factor-1 receptor in peritumoral liver tissue is associated with poor outcome in hepatocellular carcinoma after curative resection. ONCOLOGIST. 2010;15(7):732-43.

10. Y Liu, JB Zhang, Y Qin, W Wang, L Wei, Y Teng,et al. PROX1 promotes hepatocellular carcinoma metastasis by way of up-regulating hypoxia-inducible factor 1a expression and protein stability. HEPATOLOGY. 2013;58(2):692-705.

11. QH Ye, LX Qin, M Forgues, P He, JW Kim, AC Peng,et al. Predicting hepatitis B virus-positive metastatic hepatocellular carcinomas using gene expression profiling and supervised machine learning. NAT MED. 2003;9(4):416-23.

12. KJ Livak, TD Schmittgen. Analysis of relative gene expression data using real-time quantitative PCR and the 2(-Delta Delta C(T)) Method. METHODS. 2001;25(4):402-08.

13. $M$ He, W Zhang, Y Dong, L Wang, T Fang, W Tang,et al. Pro-inflammation NF-kappaB signaling triggers a positive feedback via enhancing cholesterol accumulation in liver cancer cells. J Exp Clin Cancer Res. 2017;36(1):15.

14. X Li, Q Liang, W Liu, N Zhang, L Xu, X Zhang,et al. Ras association domain family member 10 suppresses gastric cancer growth by cooperating with GSTP1 to regulate JNK/c-Jun/AP-1 pathway. ONCOGENE. 2016;35(19):2453-64.

15. J Qin, DM Gao, QF Jiang, Q Zhou, YY Kong, Y Wang,et al. Prospero-related homeobox (Prox1) is a corepressor of human liver receptor homolog-1 and suppresses the transcription of the cholesterol 7alpha-hydroxylase gene. MOL ENDOCRINOL. 2004;18(10):2424-39.

16. T Wang, P Arifoglu, Z Ronai, KD Tew. Glutathione S-transferase P1-1 (GSTP1-1) inhibits c-Jun N-terminal kinase (JNK1) signaling through interaction with the C terminus. J BIOL CHEM. 2001;276(24):209991003.

17. S Poirier, G Mayer, S Benjannet, E Bergeron, J Marcinkiewicz, N Nassoury,et al. The proprotein convertase PCSK9 induces the degradation of low density lipoprotein receptor (LDLR) and its closest family 
members VLDLR and ApoER2. J BIOL CHEM. 2008;283(4):2363-72.

18. A Demers, S Samami, B Lauzier, C Des Rosiers, ET Sock, H Ong,et al. PCSK9 Induces CD36 Degradation and Affects Long-Chain Fatty Acid Uptake and Triglyceride Metabolism in Adipocytes and in Mouse Liver. Arterioscler Thromb Vasc Biol. 2015.

19. P Labonté, S Begley, C Guévin, M Asselin, N Nassoury, G Mayer,et al. PCSK9 Impedes Hepatitis C Virus Infection In Vitro and Modulates Liver CD81 Expression. HEPATOLOGY. 2009;50(1):17-24.

20. KR Feingold, AH Moser, JK Shigenaga, SM Patzek, C Grunfeld. Inflammation stimulates the expression of PCSK9. Biochem Biophys Res Commun. 2008;374(2):341-44.

21. KR Walley, KR Thain, JA Russell, MP Reilly, NJ Meyer, JF Ferguson,et al. PCSK9 is a critical regulator of the innate immune response and septic shock outcome. SCI TRANSL MED. 2014;6(258):143r-258r.

22. H Lan, L Pang, MM Smith, D Levitan, W Ding, L Liu,et al. Proprotein convertase subtilisin/kexin type 9 (PCSK9) affects gene expression pathways beyond cholesterol metabolism in liver cells. J CELL PHYSIOL. 2010;224(1):273-81.

23. CC Mcllwain, DM Townsend, KD Tew. Glutathione S-transferase polymorphisms: cancer incidence and therapy. ONCOGENE. 2006;25(11):1639-48.

24. L Zhou, Y Jing, M Styblo, Z Chen, S Waxman. Glutathione-S-transferase pi inhibits As203-induced apoptosis in lymphoma cells: involvement of hydrogen peroxide catabolism. BLOOD. 2005;105(3):1198203.

25. MA Bogoyevitch, B Kobe. Uses for JNK: the many and varied substrates of the c-Jun N-terminal kinases. Microbiol Mol Biol Rev. 2006;70(4):1061-95.

26. RJ Davis. Signal transduction by the JNK group of MAP kinases. CELL. 2000;103(2):239-52.

27. MA Antonyak, LC Kenyon, AK Godwin, DC James, DR Emlet, I Okamoto,et al. Elevated JNK activation contributes to the pathogenesis of human brain tumors. ONCOGENE. 2002;21(33):5038-46.

28. Z Li, L Zhang, M Gao, M Han, K Liu, Z Zhang,et al. Endoplasmic reticulum stress triggers Xanthoangelolinduced protective autophagy via activation of JNK/c-Jun Axis in hepatocellular carcinoma. J Exp Clin Cancer Res. 2019;38(1):8.

29. SN Udden, YT Kwak, V Godfrey, M Khan, S Khan, N Loof,et al. NLRP12 suppresses hepatocellular carcinoma via downregulation of cJun N-terminal kinase activation in the hepatocyte. ELIFE. 2019;8:e40396

30. J Mohammad, RR Singh, C Riggle, B Haugrud, MY Abdalla, KM Reindl. JNK inhibition blocks piperlongumine-induced cell death and transcriptional activation of heme oxygenase- 1 in pancreatic cancer cells. APOPTOSIS. 2019;24(9-10):730-44.

31. V Adler, Z Yin, SY Fuchs, M Benezra, L Rosario, KD Tew,et al. Regulation of JNK signaling by GSTp. EMBO J. 1999;18(5):1321-34.

\section{Table}


Table 1. Univariate and Multivariate Analysis of Factors Associated With Overall Survival and Time To Recurrence in HCC Patients

\begin{tabular}{|c|c|c|c|c|c|c|c|c|}
\hline \multirow[t]{3}{*}{ Features } & \multicolumn{4}{|c|}{ Overal survival } & \multirow{2}{*}{\multicolumn{4}{|c|}{$\begin{array}{c}\text { Disease-free survival } \\
\text { Dival }\end{array}$}} \\
\hline & \multirow{3}{*}{$\begin{array}{l}\text { Univariate } \\
P \text { value } \\
0.202\end{array}$} & \multicolumn{3}{|c|}{ Multivariate } & & & & \\
\hline & & $\begin{array}{c}\text { Hazard } \\
\text { Ratio }\end{array}$ & $\begin{array}{l}95 \% \\
\text { CI }\end{array}$ & $\begin{array}{c}P \\
\text { value }\end{array}$ & $P$ value & $\begin{array}{l}\text { Hazard } \\
\text { Ratio }\end{array}$ & $\begin{array}{l}95 \% \\
\mathrm{CI}\end{array}$ & $\begin{array}{c}P \\
\text { value }\end{array}$ \\
\hline Age $(\leq 54$ vs. $>54$ & & & & NA & -0.242 & & & NA \\
\hline $\begin{array}{l}\text { years) } \\
\text { Gender (female }\end{array}$ & 0.905 & & & NA & 0.514 & & & NA \\
\hline $\begin{array}{l}\text { vs. male) } \\
\text { Hepatitis B }\end{array}$ & 0.776 & & & NA & 0.321 & & & NA \\
\hline $\begin{array}{l}\text { history: yes vs. no } \\
\text { HbeAg (positive }\end{array}$ & 0.159 & & & NA & 0.119 & & & NA \\
\hline $\begin{array}{l}\text { Vs. negative) } \\
\text { Liver cirrhosis: }\end{array}$ & 0.039 & 1.928 & $1.133-$ & 0.015 & 0.115 & & & NA \\
\hline $\begin{array}{l}\text { yes vs. no } \\
\text { AFP }(<400\end{array}$ & 0.000 & & 3.281 & NS & 0.000 & 0.486 & $0.275-$ & 0.013 \\
\hline $\begin{array}{l}\text { Vs. } \geq 400 \mathrm{ng} / \mathrm{ml} \text { ) } \\
\text { Preoperative ALT }\end{array}$ & 0.001 & & & NS & 0.551 & & 0.861 & NA \\
\hline BCLC stage (A vs. & 0.000 & 0.490 & $0.335-$ & 0.000 & 0.000 & 0.559 & $0.327-$ & 0.034 \\
\hline $\begin{array}{l}\mathrm{B} / \mathrm{C} / \mathrm{D}) \\
\text { Tumor } \\
\text { encapsulation (yes }\end{array}$ & 0.008 & 1.718 & $\begin{array}{l}0.717 \\
1.166- \\
2.531\end{array}$ & 0.006 & 0.035 & & & NS \\
\hline $\begin{array}{l}\text { vs. no) } \\
\text { Microvascular } \\
\text { invasion (yes vs. } \\
\text { no) }\end{array}$ & 0.171 & & & NA & 0.008 & & & NS \\
\hline Tumor & 0.710 & & & NA & 0.523 & & & NA \\
\hline VS. III/IV) & 0.000 & 0.560 & $0.335-$ & 0.027 & 0.005 & & & NS \\
\hline $\begin{array}{l}\text { PCSK9 level (low } \\
\text { vs. high) }\end{array}$ & 0.001 & 2.025 & $\begin{array}{l}1.384- \\
2.961 \\
\end{array}$ & 0.000 & 0.040 & 1.749 & $\begin{array}{l}1.034- \\
2.958 \\
\end{array}$ & 0.037 \\
\hline
\end{tabular}

\section{Figures}


Figure 1.

A.

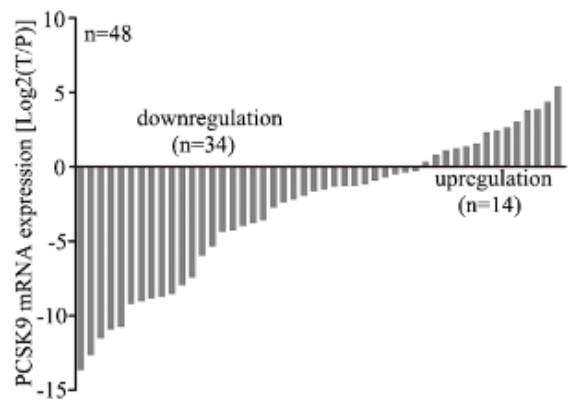

C.

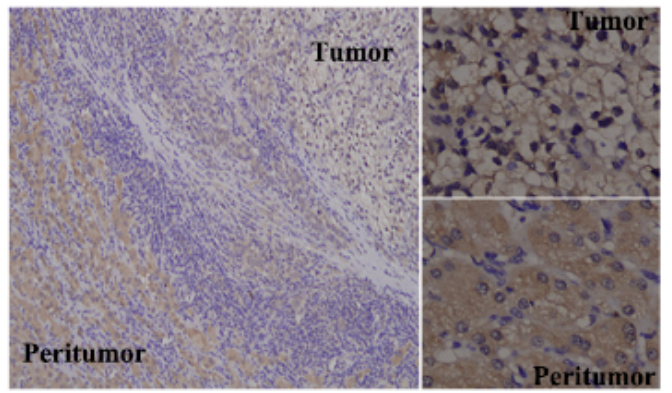

B.

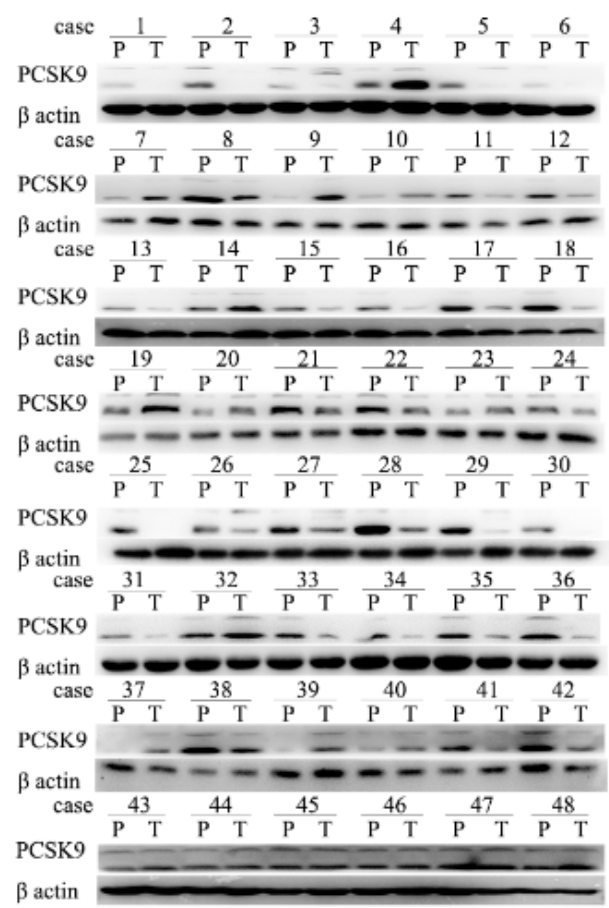

D.

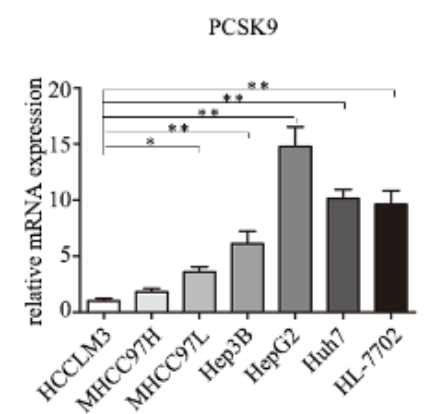

E.

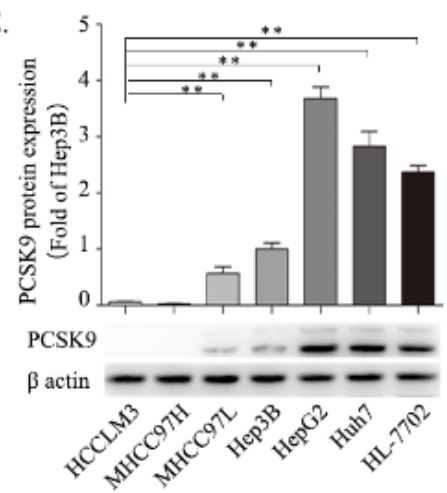

Figure 1

PCSK9 is downregulated in human HCC tissues. (A, B, C) Relative PCSK9 mRNA and protein levels were significantly decreased in HCC tissues compared with adjacent nontumor tissues $(n=48)$. (D) Relative PCSK9 mRNA levels in different HCC cell lines and the immortal normal liver cell line. (E) PCSK9 protein levels in different HCC cell lines and the immortal normal liver cell line. Data represent the mean \pm SD of triplicate samples; *P®0.05, **P®0.01. 
Figure 2

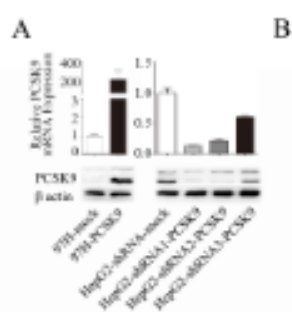

B

C
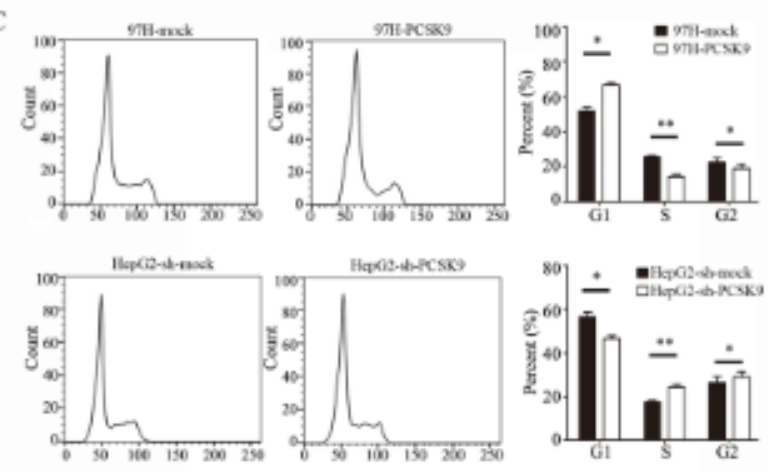

D
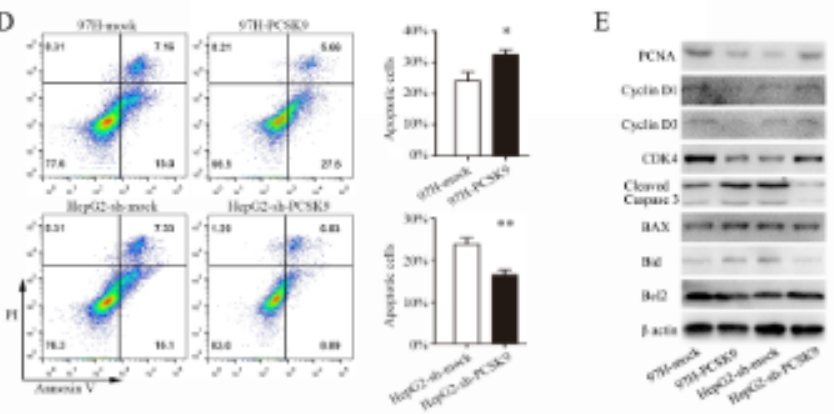

\section{Figure 2}

Effects of PCSK9 on cell proliferation, cell cycle phase distribution and apoptosis. (A) PCSK9 overexpression in $97 \mathrm{H}$ cells and successful knockdown of PCSK9 in HepG2 cells were confirmed by western blot and qRTPCR. (B) Cell growth of $97 \mathrm{H}$ and HepG2 cells was significantly changed after knockdown and overexpression of PCSK9. (C) Representative results from cell cycle analysis by flow cytometry after propidium iodide (PI) staining. (D) Representative results from apoptosis analysis by flow cytometry after dual staining by Annexin $\checkmark$ and propidium iodide (PI) staining. (E) Western blot examination showed the expression levels of proliferation markers (PCNA), apoptosis markers (Bcl-2, Bax, Bid and cleaved caspase-3) and cell cycle proteins (cyclin D1, cyclin D3, and CDK4). Data are expressed as the mean \pm SD of three independent experiments, each performed in triplicate. ${ }^{\star} p<0.05,{ }^{*} p<0.01$. 


\section{Figure 3}

A
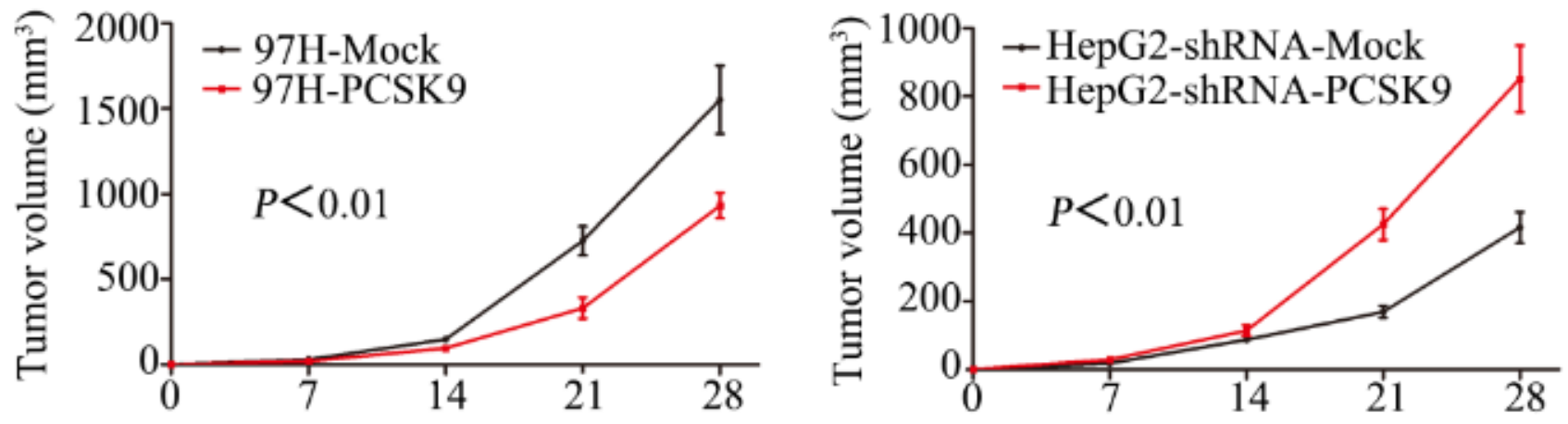

B
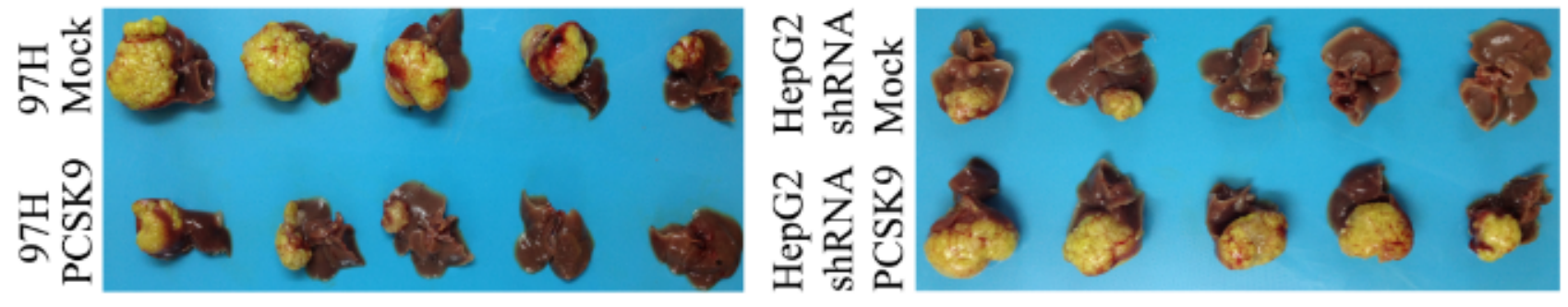

$\mathrm{C}$
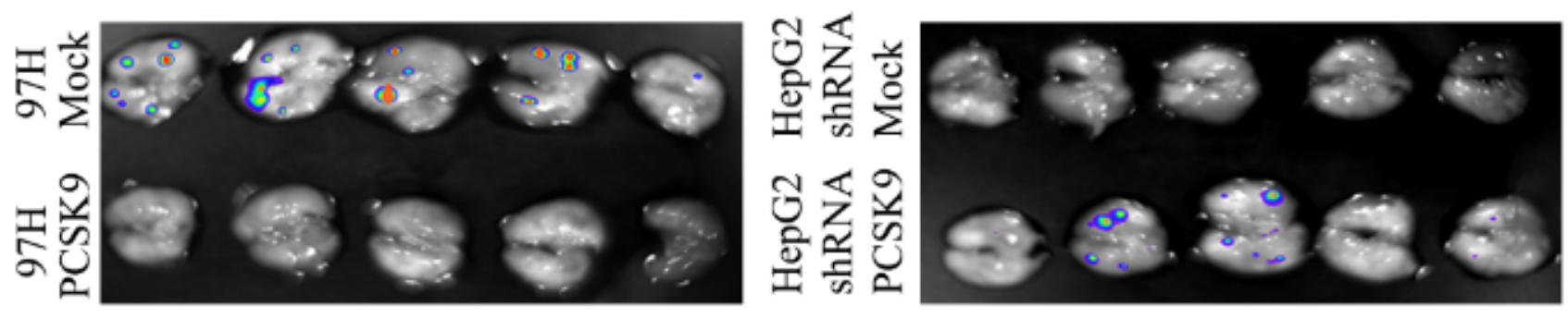

Figure 3

Effects of PCSK9 on tumor growth and metastasis of HCC in vivo. (A) The average subcutaneous tumor volumes were plotted against days. (B) Macroscopic images of isolated liver tumors of 97H-GFP and HepG2GFP. Statistical analysis using repeated-measures analysis of variance is indicated. (C) Macroscopic images of lung tissue with metastatic foci of 97H-GFP and HepG2-GFP by using an in vivo imaging system. 
Figure 4

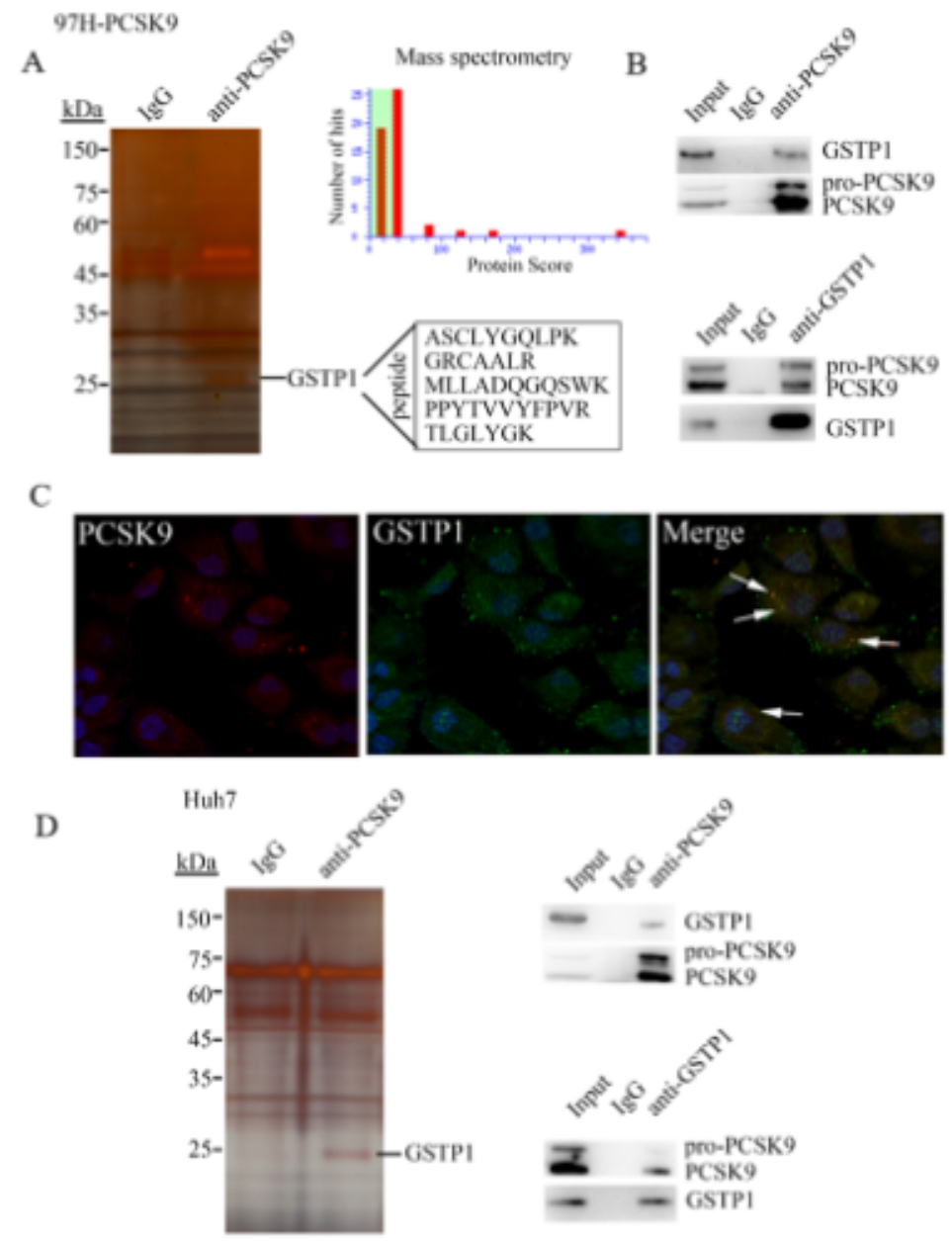

\section{Figure 4}

PCSK9 interacts with GSTP1. (A) Coimmunoprecipitation of PCSK9-binding proteins followed by mass spectrometry identified GSTP1 as a PCSK9-binding protein. (B) The presence of PCSK9 and GSTP1 in the immunoprecipitation products was confirmed by western blotting using specific antibodies. (C) Immunofluorescence staining analysis showed that PCSK9 colocalized with GSTP1 in the cytoplasm. (D) The interaction between PCSK9 and GSTP1 was further verified in Huh7 cells. 


\section{Figure 5}

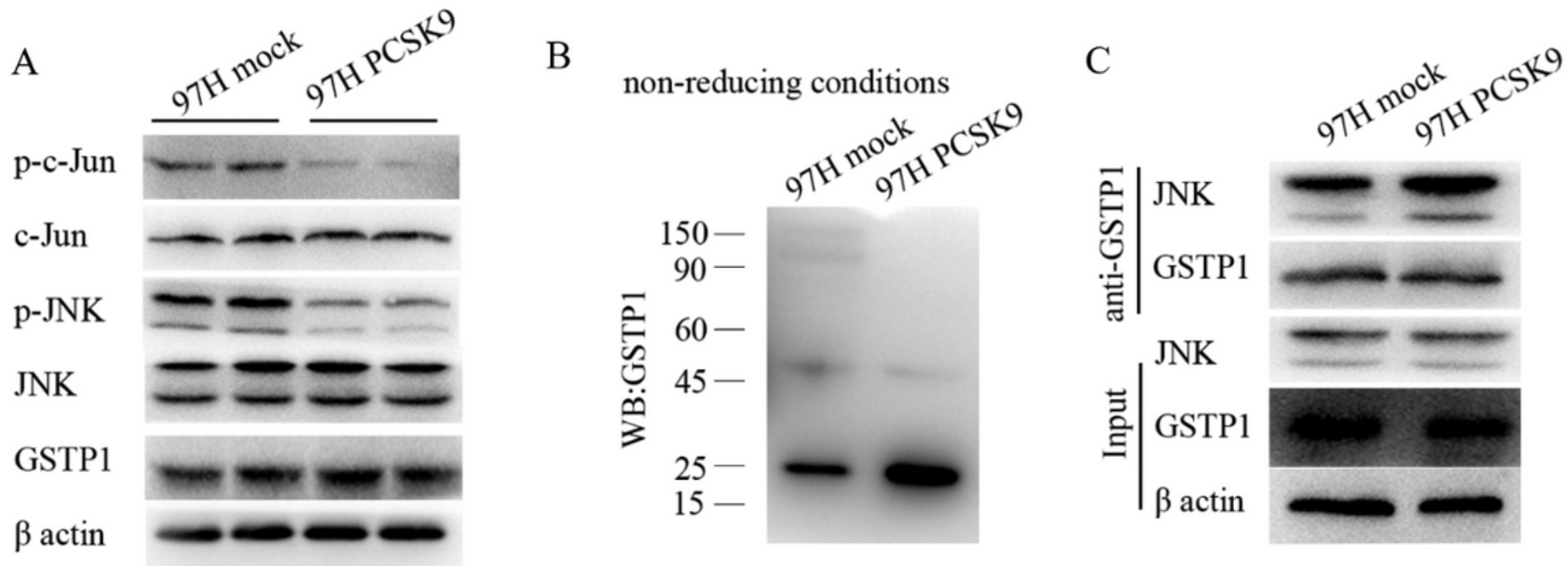

\section{Figure 5}

PCSK9 promotes the depolymerization of the GSTP1 dimer and the activity of the JNK signaling pathway. (A) The expression of GSTP1 and JNK signaling activity were detected by western blot. (B) The level of GSTP1 dimer in $97 \mathrm{H}$ cells was detected under nonreducing conditions. (C) The binding ability of GSTP1 to JNK by coimmunoprecipitation.

\section{Figure 6.}

A

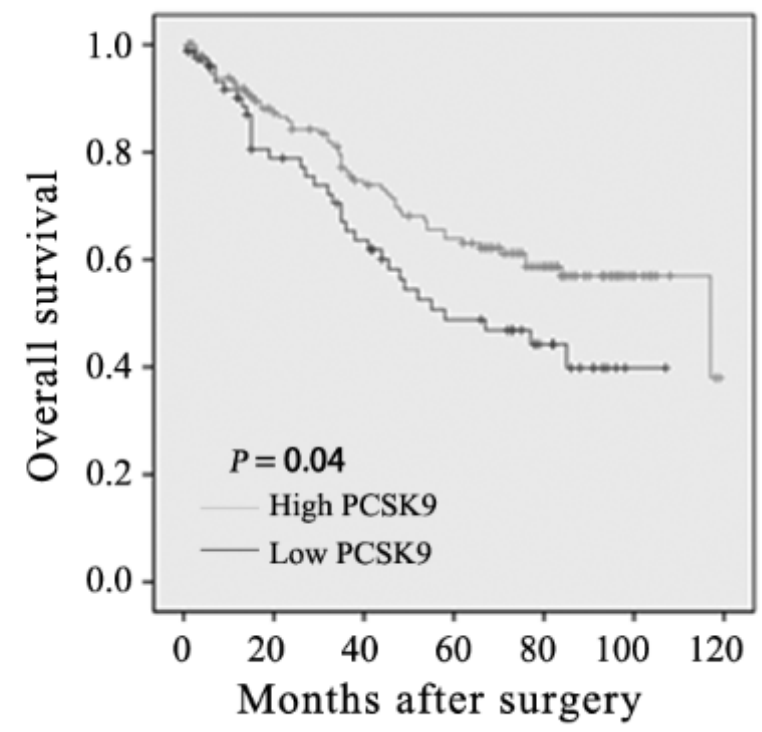

B

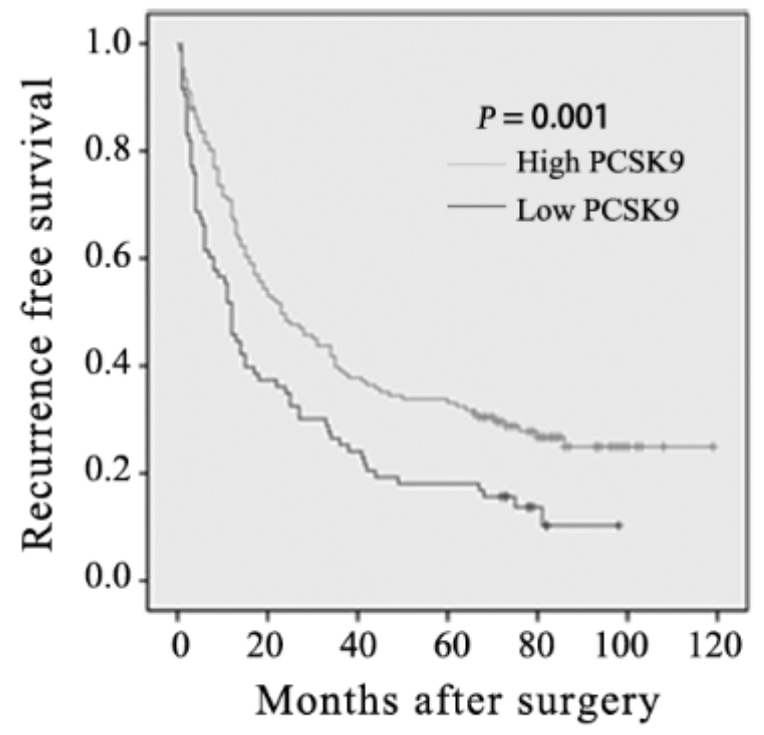

Figure 6

Correlation between the expression of PCSK9 protein and clinical outcomes. (A) Overall survival between patients with HCC in the PCSK9-hi and PCSK9-lo groups. (B) Recurrence-free survival between patients with HCC in the PCSK9-hi and PCSK9-lo groups. 


\section{Supplementary Files}

This is a list of supplementary files associated with this preprint. Click to download.

- TableS1.doc

- Tables1.doc 